\title{
THE CONSTITUTION OF
}

\section{COMETARY NUCLEI}

\author{
F. L. WHIPPLE
}

For many comets the accelerations radially from the Sun have been determined by deviations from gravitational orbits. The radial force per unit area can be calculated on the basis of certain assumptions concerning the nature of the vaporizing material, the physical circumstances, and the geometry of the cometary nucleus. The observed accelerations combined with the calculated forces yield numerical values for $(1-A) / R$ where $A$ is the albedo, and $R$ the radius of the nucleus. At extreme solar distances photometry provides the well-known guantity area-times-albedo or $R A^{l / 2}$. Solutions are then possible for $R$ and $A$ for the nuclei of the comets. The derived quantity, (1-A)A $1 / 2$, provides a limiting check on the basic assumptions and, therefore, on the basic physical properties of the nuclei. For ten short-period comets with $q<1.5 \mathrm{AU}$, the observations are satisfied by $\mathrm{H}_{2} \mathrm{O}$ ice. About half show "spotty" surfaces.

For comets of a single apparition $\mathrm{H}_{2} \mathrm{O}$ ice is generally not volatile enough to produce the observed radial accelerations. The resulting problems are discussed including the possibility that in some cases displacements of the photometric from the gravitational nucleus may produce spurious non-gravitational accelerations.

The physical characteristics of comets vary with their orbits and with their age. New comets on their first near solar passage from the Dort cloud are extremely active. The activity falls statistically with increasing age. This sequence must represent a corresponding sequence or layering of structure from the surface of a new comet inwards and is described qualitatively in this paper. The excessive activity of new comets is ascribed to cumulative cosmic-ray damage that activates the outer few hundred $\mathrm{gm} \mathrm{cm}^{-2}$ from the surface. The total

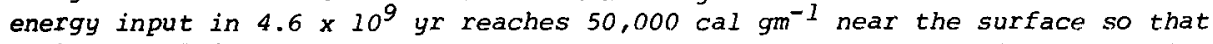
both crystalline structures and molecules are severely damaged if not completely destroyed. Annealing at $T \sim 10 \mathrm{~K}$ must be very small. Hence significant exothermic energy in the form of defects, vacancies and radicals is added to produce the extraordinary activity observed in new comets.

other aspects and problems of cometary activity are discussed.

\section{THEORY}

Let us adopt an oversimplified physical model in which we neglect reradiation from a spherical nucleus when the solar flux, F, reduced to $F(1-A)$ hy the Bond albedo, is absorbed. We shortcut also the complexities of the ClausiusClapeyron type of equation for gas production by assuming that the absorbed 


\section{WHIPPLE}

radiation is all used in vaporizing the ice, which has a latent heat, $L$, and that the gas escapes with a velocity, $v$. For a very slowly rotating nucleus the total force or pressure on the nucleus directed radially away from the Sun becomes

$$
\text { Total Force }=\pi \phi v F R^{2}(1-A) / L
$$

where $\phi$ is the double integral representing the average velocity component of the sublimed gas radially towards the Sun.

For gas production proportional to the absorbed radiation escaping normally to the surface the value of $\phi$ becomes $2 / 3$. Frequently, as here, $\phi$ is adopted as $1 / 2$, a value that Levin (1972) justly criticizes as being unrealistically large. The value may be defended when gas production is copious enough that the mean free path for the gas is small compared to $R$, that is, for active comets at fairly small solar distances. The component $\phi$ must be several times smaller than $1 / 2$ for old comet nuclei with spotty gas production. Let us adopt the notation, $\phi_{1 / 2}$ equals $\phi$ in units of $1 / 2$

The measured acceleration of the nucleus radially from the Sun, is $2 \times 10^{-5}$ $A_{1} \mathrm{~cm} \mathrm{sec}-2$ where $A_{1}$ is the radial non-gravitational term as defined hy Marsden, Sekanina and Yeomans (1973), MSY hereafter. In this notation for a solar distance, $r$, up to $r-1.5 \mathrm{AU}$, the adopted acceleration follows fairly closely the the $1 / \mathrm{r}^{2}$ law, as does $\mathrm{F}$ in $\mathrm{Eq}$. 1. The corresponding change in the gaussian constant, $k$, is closely

$$
\Delta \mathrm{k} / \mathrm{k}=0.5 \Delta \mathrm{k}^{2} / \mathrm{k}^{2}=-1.69 \times 10^{-5} \mathrm{~A}_{1} .
$$

The acceleration on a nucleus of density $\rho$ equals Eq. 1 divided hy the mass, which, when equated to the measured radial acceleration, leads to the equation for the radius as follows:

$$
\frac{R}{1-A}=\frac{15}{8} \times 10^{4} \frac{\phi_{1 / 2^{v F}}}{\rho \mathrm{LA}_{1}} .
$$

From the observed magnitude of a comet at great solar distances the quantity $R_{1}(\mathrm{~km})$ can be derived, where

$$
\mathrm{RA}^{1 / 2}=\mathrm{R}_{1} \text {. }
$$

The solar flux at $r=1 \mathrm{AU}$ is $F=1.4 \times 10^{6} \mathrm{erg} \mathrm{cm}^{-2} \mathrm{sec}^{-1}, v_{0.7}$ equals $\mathrm{v}$ in units of $0.7 \mathrm{~km} \mathrm{sec}-1, v_{0.7}=1$ being the mean velocity of $\mathrm{H}_{2} 0$, and the latent heat of vaporization of $\mathrm{H}_{2} \mathrm{O}$ is $\mathrm{L}_{6}$, in units of $640 \mathrm{cal} \mathrm{gm}^{-1}$. With these constants we find

$$
(1-A) A^{1 / 2}=\frac{R_{1} A_{1}}{0.69}\left(\frac{\rho L_{6}}{\phi_{1 / 2} v_{0.7}}\right) \text {, }
$$

where the right parenthesis equals unity for unit values of the parameters, $\rho$ in $\mathrm{gm}_{\mathrm{cm}}^{-3}$.

The albedo A can be solved as one of two solutions from Eq. 5 in terms of $A_{1} R_{1}$ and then $R$ from Eq. 4. But the quantity $(1-A) A^{1 / 2}$ sets an upper limit to $A_{1} R_{1}$ because $(1-A) A l / 2$ reaches its maximum value of 0.3849 when $A=1 / 3$. For smal 1 values of $A$ it $r i s e s$ as $A^{1 / 2}$ and at large values falls as 1 - $A$. Hence a real solution for $A$ and $R$ limits the maximum value of $R_{1} A_{1}$ :

$$
R_{1} A_{1} \leq 0.26\left(\frac{\phi 1 / 2^{v_{0}} 0.7}{\rho L_{6}}\right) \text {. }
$$


Thus for $\rho=1.3$ and the other parameters equal to unity we find the limit:

$$
R_{1} A_{1} \geq 0.20,
$$

which should be satisfied if our theory of the physical conditions in comets is realistic.

\section{APPLICATION TO SHORT-PERIOD COMETS}

A satisfactory check on the theory requires the measurement of non-gravitation radial forces for comets of small $q$ where the water-ice vaporization function, $g(r)$, used by MSY, is fairly approximated by an $r^{-2}$ law. This function is

$$
g(r)=0.1113 r_{m}^{-2.15}\left(1+r_{m}^{5.09}\right)^{-4.61},
$$

where $r_{m}=r / 2.808$ in most of the determinations.

The function $g(r)$ begins to deviate seriously from an $r^{-2}$ law beyond $r \sim 2$ $\mathrm{AU}$ and the values of $A_{1}$ determined with the $g(r)$ law are increasing too large as compared to an $\mathrm{r}^{-2}$ law for comets with $q \gg 1.0 \mathrm{AU}$. I see no simple means of allowing for this effect in applying the present theory. Thus we are limited for the moment to comets of relatively small q. Short period comets with $q<$ $1.5 \mathrm{AU}$ for which $A_{1}$ has been determined are listed in Table I in order of $q(c o l .2)$. Column 3 gives the maximum solar distance to which the comets had been observed in Kresák's (1973) compilation, mostly from measures by E. Roemer, which also lead to values of $\mathrm{R}_{1}$ (Eq. 4) in col. 5. The $\mathrm{H}_{10}$ "ahsolute" magnitudes are based on the $r^{-4}$ luminosity law from Kresák's and Vsekhsvyatski (1964).

Table I

Data for Periodic Comets with $q \leq 1.5$ AU

$\begin{array}{ccccccccc} & \mathrm{q} & \mathrm{r}_{0} & \mathrm{H}_{10} & \mathrm{R}_{1} & \mathrm{~A}_{1} & \mathrm{R}_{1} \mathrm{~A}_{1} & \mathrm{~A}^{3} & \mathrm{R} \\ \text { Comet } & \mathrm{AU} & \mathrm{AU} & \mathrm{Mag} . & \mathrm{km} & & \mathrm{km} & (\mathrm{calc})(\mathrm{km})\end{array}$

$\begin{array}{lcccccccc}\begin{array}{l}\text { Honda-Mrkos- } \\ \text { Pajdusakova }\end{array} & 0.56 & 1.2 & 11.6 & 0.48 & 0.1 & 0.05 & 0.008 & 1.2^{4} \\ \text { Giacobini-Zinner } & 0.99 & 2.5 & 11.9 & 0.70 & 0.3^{1} & 0.21 & \ldots & 1.2^{5} \\ \begin{array}{l}\text { Tuttle } \\ \text { Finlay }\end{array} & 1.02 & 2.0 & 10.0 & 2.01 & 0.32^{1} & 0.64 & \ldots & 3.5^{5} \\ \begin{array}{l}\text { Tutt1e-Giacobini- } \\ \text { Kresak }\end{array} & 1.08 & 2.0 & 12.5 & 0.26 & 0.5^{1} & 0.13 & 0.07 & 0.7^{4} \\ \text { D'Arrest } & 1.15 & 1.7 & 13.9 & 0.14 & 0.66^{2} & 0.09 & 0.033 & 0.4^{4} \\ \text { Schaumasse } & 1.17 & 2.8 & 12.0 & 0.28 & 0.8^{2} & 0.22 & \ldots & 0.5^{5} \\ \text { Tempel 2 } & 1.20 & 2.8 & 12.0 & 0.88 & 0.4^{2} & 0.35 & \ldots & 1.5^{5} \\ \text { Jackson-Neujmin } & 1.37 & 3.6 & 10.4 & 0.53 & 0.1^{1} & 0.05 & 0.010 & 1.4^{4} \\ \text { Borre1ey } & 1.43 & 1.9 & 15 ? & 0.20 & 0.8 & 0.16 & 0.12 & 0.5^{4} \\ & 1.45 & 3.0 & 12.5 & 0.66 & 0.09 & 0.06 & 0.013 & 1.7^{4}\end{array}$

1 Maximum value if variable

Most recent value

Lower value of A adopted, $\rho=1.3$

A adopted as 0.15 , in $\mathrm{Eq} .4$

$\mathrm{A}$ adopted as $1 / 3$, in $\mathrm{Eq} \cdot 4$ 
The subsequent columns are, respectively, $A_{1}$ from Marsden et al., $R_{1} A_{1}$ calculated from cols. 5 and 6 , A by Eq. 5 with $\rho=1.3$, and, $R$, the cometary radius calculated from Eq. 4 with $A=1 / 3$ or 0.15 as indicated.

Table I shows that of the ten examples, only P/Tuttle deviates seriously from the condition $R_{1} A_{1} \leq 0.20$, giving $R_{1} A_{1}=0.64$. But MSY find that $A_{1}$ for $P / T u t t l e$ has been essentially zero in the interval 1912-1967 and so the discrepancy appears not to be real. There are also 11 comets of period less than $20 \mathrm{yr}$. for which MSY find no measurable radial accelerations $\left(A_{1}\right)$ and for which Kresák finds $R_{1}<0.9$. We may therefore conclude that our crude theory applies well to short-period comets, particularly to those with $q<1.5 \mathrm{AU}$.

The very small values of Albedo calculated for the six comets that completely satisfy the theory indicate clearly that these comets must be spotty, that is, mostly covered with meteoroidal material that does not vaporize readily. This is almost certainly true also for P/D'Arrest because the derived values of $A_{1}$ have been quite variable, dropping essentially to zero at times. The same is probably true for $P / G i a c o b i n i-Z i n n e r$ as $A_{1}=0.3$ is a relatively large value for this comet (MSY).

Conclusion: Activity of short-period comets with small q is consistent with control by $\mathrm{H}_{2} \mathrm{O}$ ice, in some cases only partially covering their surfaces.

\section{LONG-PERIOD COMETS}

Delsemme and Rud (1973) have derived the albedo and radius for C/Bennett 1970 II. $\mathrm{RA}^{1 / 2}=\mathrm{R}_{1}$ (Eq. 4) is given by limiting observed magnitude, and the observed production rates of $\mathrm{H}$ and $\mathrm{OH}$ are assumed to be derived from $\mathrm{H}_{2} \mathrm{O}$ ice. The later measures, coupled with an assumed latent heat of vaporization, $\mathrm{L}_{6}=1$, establishes a numerical value of $\pi R^{2}(1-A)$. They applied this method also to C/Tago-Sato-Kosaka, 1969IX, and O'Dell (1976) has applied it to C/Kohoutek, 1973 XII. Neither of these latter comets, however, shows non-gravitational forces and so $\mathrm{C} /$ Bennett remains the only test case for comparing the methods on long-period comets. Because $q=0.54$ is small the proposed methgd should apply.

The observed constants for $C /$ Bennett are $\pi R^{2}(1-A)=15.7 \mathrm{~km}^{2}, R_{1}=3.05 \mathrm{~km}$ and $A_{1}=1.9 \pm 0.1$ (MSY). We immediately note that $R_{1} A_{1}=5.8$, compared to our upper limit (Eq. 7) of 0.20 , a discrepancy of 29 times.

The theories cannot be reconciled simply by an assumed change in the heat of vaporization because the value of $\pi R^{2}(1-A)$ in the Delsemme-Rud method and $(1-\mathrm{A}) \mathrm{A}^{1 / 2}$ in the present method (Eq. 5) are both proportional to $\mathrm{L}_{6}$, so that $\mathrm{L}$ cancels out in combining the three observed quantities. The solution leads to $A \gg 1$. Changes of more than an order of magnitude (Eq. 5) in the mean density $\rho$ (to $\sim 0.05 \mathrm{gm} \mathrm{cm}^{-3}$ ), in the velocity of ejection or in the geometrical constant $\phi$, cannot be considered seriously. An attempt to gain consistency by placing the blame on an unobserved halo about the comet when E. Roemer last observed it at $\mathrm{r}=4.5 \mathrm{AU}$ also leads to an inconsistency. The necessary correction to $R_{1}$ becomes imaginary and requires $A \cong-3$. We must conclude that the theory requires modification in more than one parameter or else that the non-gravitational force is overestimated.

Application of the present theory to several single-apparition long-period or new comets leads to values of $R_{1} A_{1}$ that also exceed the $\mathrm{H}_{2} \mathrm{O}$-ice 1 imit of 0.20 by a large factor. The deviation might be explained on the assumption that the non-gravitational radial forces are in error. Suppose, for example, that the center of the measured nucleus is displaced systematically from the physical center (note Debehogne 1968). If the apparent displacement is radial towards the Sun and equal to $\varepsilon r$, it can be shown that the resultant orbit follows Newtonian motion perfectly when the gaussian constant, $k$, is corrected by $\Delta k$ where

$$
\Delta \mathrm{k}^{2} / \mathrm{k}^{2}=\Delta\left(\mathrm{GM}_{\mathrm{o}}\right) / \mathrm{GM}_{\mathrm{o}}=-3 \varepsilon \text {, }
$$


in which $G$ is the constant of gravitation and $M_{0}$ the mass of the Sun (note Eq. 2).

For $C /$ Bennett $I$ find that the equivalent displacements to make $A_{1}=1.9$ range from 1!4 to $3: 7$ as seen from the Earth, and, including geocentric distance, projected transverse distances at the comet ranging from 1700 to $3300 \mathrm{~km}$, irregularly distributed over the orbit. It is not possible without a much more thorough analysis to deduce whether smaller displacements suitably placed might not have produced a similar result.

Of great relevance are the observations of $C /$ Bennett by Malaise (1976) who measured relative displacements from the nucleus towards and away from the Sun, in the light from various important radicals. He found projected displacements amounting to $1000 \mathrm{~km}$ and more. I emphasize the need for such detailed measurements of apparent nuclear displacements for other comets in order to determine the extent to which the orbit calculations may be affected. Only then, for these comets, can we be more assured as to the reality of radial non-gravitational calculations.

On the other hand, I find that the new comet Burnham, 1960II, with $A_{1}=$ 5.8 , (MSY) would have required much larger displacements, well outside the permissible image diameters, to have given a completely spurious value of $A_{l}$. From a tentative study of the actual circumstances of a few comets I am convinced that the measured radial non-gravitational forces are real for some, at least, of the several comets for which MSY have determined them.

That the inclusion of the radial non-gravitational forces in orbit determinations for new comets reduces the calculated hyperbolicity is a strong argument advanced by MSY in support of the reality of the forces. Another argument for the reality is the persistence of positive derived values of $A_{1}$, indicating negative values of $\Delta \mathrm{k} / \mathrm{k}$ and hence forces on comet nuclei directed radially away from the Sun.

\section{THE INTERNAL STRUCTURE OF COMETS}

We have seen that short-period comets of small $q$ are activated by the sublimation of $\mathrm{H}_{2} \mathrm{O}$ ice, or show an equivalent reaction. Were this the only ice in comets, the activity would be essentially nil beyond $r \sim 2.5$ to $3.2 \mathrm{AU}$. But some comets have shown violent activity at far greater solar distances. Individual differences are striking, particularly among "new" comets. Hence we may adopt the working hypothesis of structural or compositional changes with depth among comets, a layered structure within a pristine new comet. Among comets of various ages, measured by some function combining surface mass loss rates and perihelion passages, we are observing layers of different icy compositions or structures.

The following picture of a comet then emerges from the studies and speculations of many contributors (e.g., Levin, this volume, Opik 1963; Shul'man 1972a; Sekanina 1976):

1. The Outer Frosting. This layer is thin, extending to a mass depth of only a few hundred $\mathrm{gm}_{\mathrm{cm}}^{-2}$, and appears on most new comets. The ices are extraordinarily volatile and probably extremely porous. Often the frosting is removed in the progress of the first perihelion passage of a new comet. Possibly some small comets consist only of frosting and are very short lived. Probably the dust/gas ratio is higher in the frosting than in the interior and possibly $\mathrm{H}_{2} \mathrm{O}$ and therefore $\mathrm{O}$ is depleted compared to $\mathrm{C}$ (and $\mathrm{N}$ ) compounds (see Delsemme, this volume).

Strong evidence supporting the frosting concept is Marsden and Sekanina's (1973) observation that comets of large perihelion distance include such a large fraction of new comets. Many old comets should be expected except for the selection effect of our telescopes. We observe only the very brightest so that a loss of perhaps two magnitudes on the first apparition by the removal of the 
frosting has rendered such comets unobservable on subsequent returns. Marsden and Sekanina discuss other evidence supporting such a supposition.

2. The Upper Mantle. Most "youthful" comets present us with the upper mantle layer, which is highly variable from comet to comet but is much more volatile than $\mathrm{H}_{2} \mathrm{O}$ ice. Representatives are probably $\mathrm{C} /$ Bennett and many of the shorter period comets of large $q$ and those that have recently been brought in closer to the Sun by Jupiter. The mantle probably contains the original $\mathrm{H}_{2} \mathrm{O}$ composition plus much material that is far more volatile. In adjusting to a reduced perihelion distance the comet is more active, as Kresaik (1973) emphasizes, until the outermost layer is stratified with depth as follows: dust, $\mathrm{H}_{2} \mathrm{O}$ ice, and finally the average mantle composition.

The outbursts accompanying the splitting of comets demonstrates that the surface stratification by solar heating covers an interior of far greater volatility.

The "dustiness" of the mantle varies from comet to comet but may only represent particle-size distribution variations, not variations in the total dust to ice ratio.

3. The Lower Mantle, or the $\mathrm{H}_{2} \mathrm{O}$ Ice Meteoroidal Layer. Most of the shortperiod comets of small perihelion distance present this lower part of the mantle. Among these older comets the material is more consolidated so that activity is progressively more inhibited by cohesion. The surface stratification produced by solar heating becomes so pronounced with age that many areas remain inactive much of the time. Hence the surface activity is highly spotted, whether or not the cometary mass contains irregular volumes or pockets of different compositions -- the raisin-cake model. Probably some of the more volatile ices have been transported outward by mild (radioactive?) heating (Whipple and Stefanik 1966). These comets usually do not appear dusty, probably because the meteoroidal material is better cemented and is removed in larger pieces, ineffective in scattering sunlight. The dust/gas ratio may well increase with depth throughout the mantle.

4. The core. Some investigators would call this region the "asteroidal". core, totally (?) inactive insofar as cometary activity is concerned. Whether such cores contribute significantly to the asteroid population, particularly those with perihelia near or within the Earth's orbit, remains unproven. Possibly only extraordinarily large comets have cores. We do not know whether the cores contain $\mathrm{H}_{2} \mathrm{O}$ ice and may be inactive only because the cohesion of the crust prevents the ejection of solids and inhibits heat flow to the interior. Thus we have no evidence to prove whether the cores arose by internal heating that transferred the ices to the outer layers or whether the cores represent meteoroidal fundations on which the comets grew by accretion.

Generally the activity of a comet decreases with increasing depth from the outer mantle to the core. How much of this change in activity arises from increasing cohesion of the meteoroidal material with depth and how much because of a decrease in the fraction of volatiles remains an open question. Probably transfer of volatiles outward by radioactive heating plays a role (Whipple and Stefanik). Probab1y, also, cosmic rays play a role in the outer part of the outer mantle.

Because of limitations of space I shall not further elaborate the physical properties of the inner regions of comets but concentrate on a possible cause for the extraordinary properties of the cometary frosting and for the vagaries among the "younger" comets.

\section{ON THE NATURE AND CAUSE OF THE FROSTING}

Present evidence points strongly to the origin of comets simultaneously with the solar system generally, some $4.6 \times 10^{9} \mathrm{yr}$ ago. On this basis the surfaces of comets in the Oort cloud have been exposed to cosmic rays for this 


\section{CONSTITUTION OF COMETARY NUCLEI}

enormous period of time. The exposure rate has presumably been fairly constant except for possible brief excursions upwards because of supernovae and perhaps downwards within dense interstellar clouds. Accretion of interstellar dust has probably been negligible, much less than $1 \mathrm{gm} \mathrm{cm}^{-2}$.

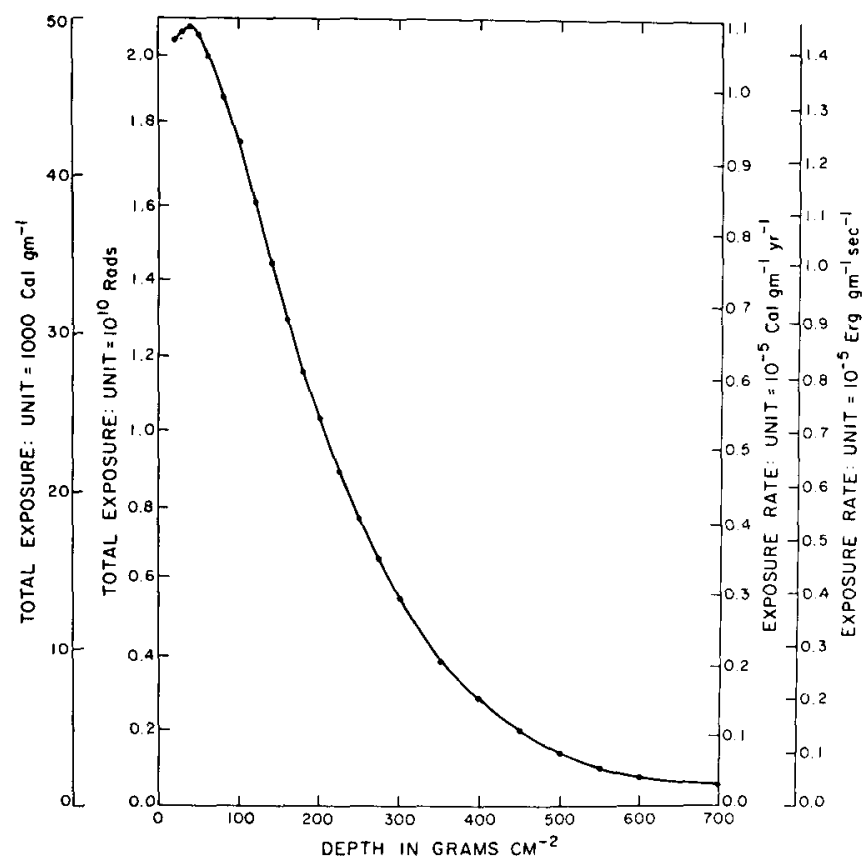

Figure 1. Ionizing Exposure by Cosmic Rays in Comets as Function of Mass Depth: total exposure over $4.6 \times 10^{9}$ yr (left) and exposure rate (right), based on Bowen, Millikan and Neher.

The cumulative total energy from cosmic rays deposited as ions of $\sim 30 \mathrm{ev}$ in the surface layers of comets over a period of $4.6 \times 10^{9}$ years is shown in Fig. 1, extrapolated from balloon measures by Bowen, Millikan and Neher (1938).

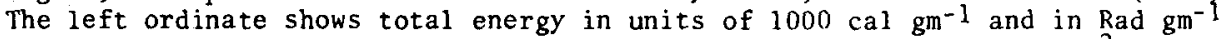
( $1 \mathrm{Rad}=100 \mathrm{erg}$ ) versus the depth from the cometary surface in $\mathrm{gm} \mathrm{cm}^{-2}$ as abscissa. The right ordinate gives the rate in units of $10^{-5} \mathrm{cal} \mathrm{gm}^{-1} \mathrm{yr}^{-1}$ and $10^{-5} \mathrm{erg} \mathrm{gm}^{-1} \mathrm{sec}^{-1}$.

Figure 1 shows that the upper surface layers of new comets have received more than 50 times the heat of vaporization of $\mathrm{H}_{2} \mathrm{O}$ ice. At a depth of $400 \mathrm{gm}$ $\mathrm{cm}^{-2}$ the total cosmic-ray energy contribution is still 10 times that quantity. The small input energy rates on the right ordinates show, however, that heating accompanied by ordinary vaporization should not have occurred. The heat can readily be radiated away to space at temperatures $<20 \mathrm{~K}$ (Whipple and Stefanik, Fig. 2) even for comets of $R \sim 100 \mathrm{~km}$ with such energy inputs throughout.

Berger (1961), Shul'man (1972a) and Donn (1976) have recognized the huge ionizing input of cosmic rays to comets, generally consistent with the data in Fig. 1. Donn concludes that "the irradiation will tend to polymerize the simple, volatile original ices," thereby reducing the surface activity of new comets. Shul'man, on the other hand, suggests that galactic cosmic rays might produce complicated molecules such as $\mathrm{C}_{3} \mathrm{H}_{4}, \mathrm{CH}_{2} \mathrm{~N}_{2}, \mathrm{C}_{4} \mathrm{H}_{2}$ and others from simpler com- 
pounds of $\mathrm{H}, \mathrm{C}, \mathrm{N}$ and $\mathrm{O}$. He also suggests that cosmic rays from solar flares might activate the extreme outer layers of comets (mm's ?) by "radiation synthesis and accumulation of unsaturated hydrocarbons and other explosives " to produce the energy source for cometary bursts in luminosity. Berger's experiment with approximately equal masses of $\mathrm{CH}_{4}, \mathrm{NH}_{3}$ and $\mathrm{H}_{2} \mathrm{O}$ irradiated at $77^{\circ} \mathrm{K}$ with 12 mev protons shows that acetone and complex hydrocarbons are produced.

The important effect of cosmic rays is clearly to alter or destroy all molecular structures to the saturation limit at depths to several $100 \mathrm{gm} \mathrm{cm}^{-2}$. The more energetic cosmic rays can produce less serious damage to depths of perhaps $10^{4} \mathrm{gm} \mathrm{cm}^{-2}$. Dienes and Vineyard (1957) and Chadderton and Torrens (1969) summarize a great deal of evidence relevant to crystalline-structure damage. They report that reactor damage to graphite at $-190^{\circ}$ tends to anneal out at room temperature. But in a furnace at $200^{\circ} \mathrm{C}$ the graphite can give out as much as 50 cal $\mathrm{gm}^{-1}$ from the radiation damaged crystalline structure.

Near the surface of a new comet the cosmic rays produce nearly one ion per nucleon in $4.6 \times 10^{9} \mathrm{yr}$. and, therefore, a number of ions per molecule. The damage to any crystalline structure must be total and complete restructuring of the molecules must occur. The annealing process at $\sim 10 \mathrm{~K}$ must be negliglble after the immediate interactions $\left(-10^{-11} \mathrm{sec}\right)$ are over. The phenomena of radiation damage are extremely complicated but for cometary material one might expect not only the usual lattice displacement of atoms and lattice defects in crystals, but also the production of radicals from the molecules. Furthermore, some loss of mobile atoms such as $H$ should occur and a considerable amount of chemical transformation take place, ending in amorphous material, with considerable free energy available upon heating.

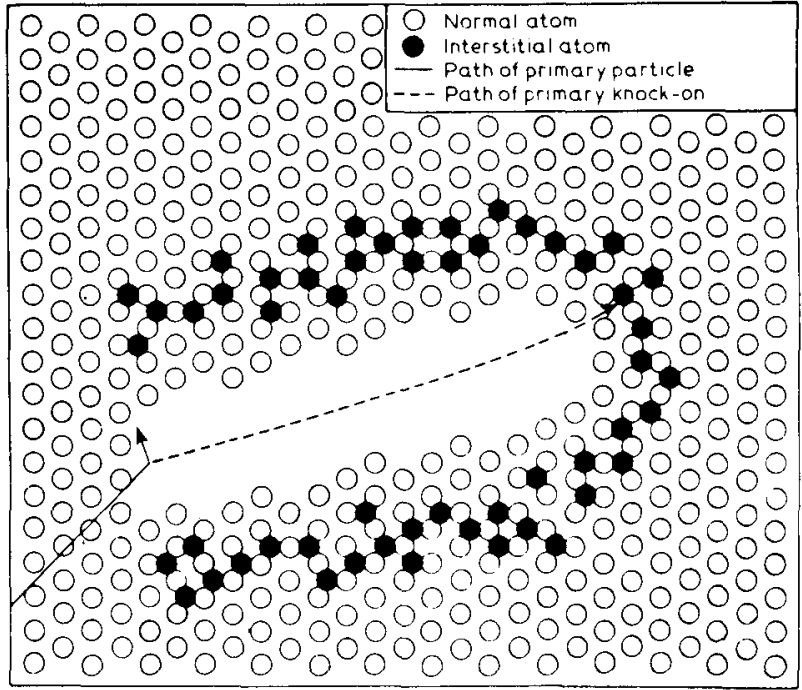

Figure 2. Brinkman Displacement Spike of High-Energy Fragment in Crystal. (From Chadderton and Torrens).

The more detailed nature of the interactions can be visualized by the displacement spike concept originated by J. A. Brinkman and illustrated in Fig. 2. As Chadderton and Torrens describe the process, a multiple vacancy in the lattice structure should be created at the end of the path of a fission fragment of high energy or an energetic primary knock-on atom. Interstitial atoms are knocked into the lattice. The temperature should be very high $\left(-1000^{\circ}\right)$ momen- 
tarily, and considerable migration of atoms to interstitial positions should occur, followed by partial collapse of the spike and only partial annealing. Dienes and Vineyard calculate that a $300 \mathrm{ev}$ thermal spike in copper should raise the temperature to the melting point over a 30 \& diameter. Lengths of the spikes can reach hundreds of angstroms branching out from the path of the primary particle.

The displacement and thermal spikes will further disorder the atoms in a comet, which are probably already a chaotic chemical mix. The spikes, by their atomic displacements, may also tend to join together adjacent dust particles if the grains are some $0.1 \mu$ or $10^{3} \AA$ in dimension, thus producing clumps of grains that are extremely weak structurally. Thus the radiation damaged surface of a new comet, weakly heated by solar radiation at great distances, may augment the sublimation of highly volatile materials by exothermic reactions that expel gas and grain clumps. This may explain the existence of distant diffuse comae, seen for example about $\mathrm{C} /$ Kohoutek, $1973 \mathrm{XII}$ at discovery, $\mathrm{r}=4.7 \mathrm{AU}$.

Although the conclusions of this paper are somewhat speculative in the sense that the precise physical mechanisms and the exact phenomenological consequences remain only partially explored, these concepts lead to even more speculative questions that deserve careful consideration both in laboratory experiments and in theoretical developments. Among these are the following:

a) Can cosmic-ray damage and radicals create cometary materials in which exothermic reactions can occur as a result of only moderate solar heating at great solar distances? In other words, can cometary outbursts be attributed to such intrinsic energy sources in comets?

b) Is it possible that some comets such as P/Schwassmann-Wachmann I contain a layer of intrinsically active material in which small volumes are occasionally set off to produce the fairly frequent outbursts that characterizes the comet? Is it possible that a supernova occurring in the interstellar cloud of the protosolar system produced layers of intrinsically active material on large comets, which were covered by ordinary comet material and now irregularly exposed in such comets?

c) Can near saturation levels of radiation damage and radical production occur in comets over periods of a few million years so that comets of very long period actually accumulate appreciable energy during their long excursions into deep space? This might account for unusual activity in comets with extremely long period.

d) Do the more energetic cosmic rays penetrating to depths of $10^{3}-10^{4}$ $\mathrm{gm} \mathrm{cm}$ in the upper mantle provide some intrinsic energy for the longer period comets and possibly tend to bind the dust of the upper mantle into larger aggregates?

e) It is possible that high cosmic-ray concentration in the region of the solar system during its formation has added radiation damage and radical energy to the entire mantles of comets? $O r$ is it possible the solar wind and solar flares during the Hayashi period of the Sun's formation made such contributions to the then forming comets?

These questions are all in addition to the more classical ones concerning the uncertainty with regard to intrinsic radioactive materials imbedded in the cores of comets which may have produced enough heat to transfer the ices from the cores to the mantles. A great deal depends upon the quantity of short-lived radioative sources such as $26_{\mathrm{Al}}$ that may have contributed substantial heat during and immediately after comet formation. Can the transport of volatiles outwards and their freezing near the surface produce sealed volumes of gas and/or exothermic materials that "explode" on mild solar heating to produce comet outbursts?

Finally there remains the major question as to whether the decreasing cometary activity from the upper mantle to the core arises from a systematic 
change in composition and volatility or simply from an increase in the cohesiveness of the meteoroidal material.

I am particularly indebted to Edward L. Fireman for advising and assisting me in assembling the cosmic-ray data. Also the paper has benefited from many discussions with Brian G. Marsden and Zdenek Sekanina and the use of some of their unpublished data.

This study was partially supported by Grant No. NSG 7082 from the National Aeronautics and Space Administration.

\section{REFERENCES}

Berger, R. 1961, Proc. Nat. Acad. Sci., 47, 1434.

Bowen, I. S., Millikan, R. A., and Neher, H. V. 1938, Phys. Rev. 53, 855-861. Chadderton, L. T., and Torrens, I. M. 1969, Fission Damage in Crystals, Halsted Press.

Debehogne, H. 1968, Bull. d. Sci., Acad. Roy. Belgique, Ser. 5, 14, 1040-1045. Delsemme, A. H., and Rud, D. A. 1973, Astr. Astrophys., 28, 1.

Diens, G. J., and Vineyard, G. H. 1957, Radiation Effects in Solids, Interscience, N.Y.

Donn, B. 1976, The Study of comets, NASA SP -393, 611-619,

Kresák; L. 1973, Bu11. Astr. Inst. Czech., 24, 264-283.

Levin, B. Yu. 1972, IAU Symp., No. 45, D. Reidel Publ. Co., Dordrecht-Holland, p. 260 .

Malaise, D. 1976, The Study of Comets, NASA SP-393, 740-749.

Marsden, B. G. 1975, Catalogue of Cometary Oribts, Smith. Astrophys. Obs., Cambridge.

Marsden, B. G., Sekanina, Z., and Yeomans, D. K. 1973, Astr. J., 78, 211.

Öpik, E. J. 1963, Advances in Astronomy and Astrophysics, vol. 2, ed. 2. Kopal, Academic Press, p. 219.

Sekanina, Z, 1976, The Study of Comets, NASA SP-393, 537-588.

Shul'man, L. M. 1972a, The Motion, Evolution of Orbits and the Origin of comets, IAU Symp. No. 45, 260.

Vsekhsvyatski, S. K. 1964, Physical Characteristics of Comets, Translation Israel Program, NASA TT F-80.

Whipple, F. L., and Stefanik, R. P. 1966, Mem. Soc. Roy. Liege, Ser. 5, Vol. I2, 33-52.

\section{DISCUSSION}

DONN: Some years ago Dr. Jackson and I calculated the photochemical production of radicals and obtained density contours. The maximum was displaced in the solar direction by amounts depending upon the heliocentric distance and comet luminosity. For bright comets coming in close, the displacement may be several thousand kilometers. It will be less for periodic comets than for Bennett. This effort is in the direction you were proposing.

A second point: At the Goddard Colloquium I made similar calculations of cosmic ray irradiation and chemical effects produced. The large amount of irradiation is enough to completely rework the chemical composition. I concluded that the high radical concentration, even at low temperature would produce complex organic material of low volatility. That presents a dilemma. A way out may be the presence of an inert volatile component. Argon, with a cosmic abundance I would estimate comparable to $\mathrm{CO}$ or $\mathrm{CO}_{2}$ concentrations in comets, may be 


\section{CONSTITUTION OF COMETARY NUCLEI}

such a material. Finally, the polymerized, irradiated surface would probably be a dark material with a very low albedo. This would make the sunlight act more efficiently on new comets.

WHIPPLE: I apologize for not registering in my mind your comments on cosmic rays. I question that polymerization would occur in such a heterogenous material, but lowering of albedo would be a very interesting possibility. 